\title{
Gastric Cancer Cells in Peritoneal Lavage Fluid: A Systematic Review Comparing Cytological with Molecular Detection for Diagnosis of Peritoneal Metastases and Prediction of Peritoneal Recurrences
}

\author{
EDOARDO VIRGILIO $^{1}$, ENRICO GIARNIERI ${ }^{2}$, MARIA ROSARIA GIOVAGNOLI $^{2}$, \\ MONICA MONTAGNINI ${ }^{2}$, ANTONELLA PROIETTI $^{2}$, ROSARIA D'URSO $^{2}$, PAOLO MERCANTINI $^{1}$, \\ STEFANO VALABREGA ${ }^{1}$, GENOVEFFA BALDUCCI ${ }^{1}$ and MARCO CAVALLINI ${ }^{1}$
}

Departments of ${ }^{1}$ Medical and Surgical Sciences and Translational Medicine, and ${ }^{2}$ Clinical and Molecular Medicine, Faculty of Medicine and Psychology, Sapienza University, St. Andrea Hospital, Rome, Italy

\begin{abstract}
Background/Aim: Detecting free tumor cells in the peritoneal lavage fluid of gastric cancer patients permits to assess a more accurate prognosis, predict peritoneal recurrence and select cases for a more aggressive treatment. Currently, cytology and molecular biology comprise the two most popular methods of detection that are under constant study by researchers. Materials and Methods: We burrowed into the available literature comparing cytological with molecular detection of free intraperitoneal gastric cancer cells. PubMed, Science Direct, Scopus and Google Scholar were the search engines investigated. Results: As of 2017, 51 dedicated studies have been published. Messenger RNA of carcinoembryonic antigen was the genetic target most frequently described. The genetic technique is usually superior to cytology in sensitivity (38-100\% vs. 12.3-67\% respectively), whereas cytological examination tends to show a slight preeminence in specificity (approximately 100\%). Conclusion: So far, given the imperfection of each method, employment of both cytology and molecular examination seem to be mandatory.
\end{abstract}

This article is freely accessible online.

Correspondence to: Dr. Edoardo Virgilio, Department of Medical and Surgical Sciences and Translational Medicine, Division of General Surgery, Faculty of Medicine and Psychology, University "Sapienza", St. Andrea Hospital, via di Grottarossa 1035-39, Rome 00189, Italy. Tel: +39 0633775989, Fax: +39 0633775322, e-mail: aresedo1992@yahoo.it, edoardo.virgilio@uniroma1.it

Key Words: Gastric cancer, peritoneal lavage, peritoneal carcinomatosis, peritoneal recurrence, cytopathology, molecular detection, RT-PCR, review.
Currently, despite the amelioration and standardization of surgery techniques and multi-modal therapy, the prognosis of gastric cancer (GC), especially that of advanced GC (AGC) with serosal invasion (T3 or T4 cancers), remains very poor with a 5-year overall survival (OS) of less than $35 \%$ (1). Peritoneal dissemination is the most common route of metastasis followed by AGC and leads to peritoneal recurrence (PR) which is the most frequent cause of death (up to $60 \%$ of cases within 2 years) even if curative resection is performed (2). Hence, in the case of AGC, detecting intraperitoneal free cancer cells is of paramount importance because it is significantly related to the prediction of peritoneal metastasis (PM) and patients' prognosis $(1,2)$. Accordingly, since 1998 the Japanese Gastric Cancer Association (JGCA) recommends to perform peritoneal lavage cytology (PLC) to detect free floating malignant cells within the peritoneal cavity; in addition, in 2010, a positive PLC was classified as metastatic disease also in the 7 th edition of the American Joint Committee on Cancer (AJCC) tumor node metastasis (TNM) staging system for GC (3). However, despite its high specificity, conventional PLC shows a questionable sensitivity (11.1 to $80 \%)$ : in fact, cytology-negative cases often develop PR and meet with worse prognosis $(1,2)$. In an effort to enhance sensitivity, in the last decades researches have focused on the detection of several epithelial cell-related targets using molecular biology methods. Herein, we offer a meticulous review of the knowledge and progress so far achieved in terms of diagnostic and prognostic results through cytological and genetic examinations of peritoneal lavage (PL) in patients affected with AGC. 


\section{Materials and Methods}

We systematically reviewed the world literature dealing with the detection of free intraperitoneal cancer cells and comparing cytopathological with molecular examination of peritoneal lavage fluid obtained from patients with GC and AGC. The investigation was carried out through four popular search engines (PubMed, Science Direct, Scopus and Google Scholar). GC, AGC, PC, PM, PLC, genetic detection, molecular diagnosis and Real time reverse chained transcriptase-polymerase chain reaction (RT-PCR) were the key words utilized for searching. Only works comparing the two aforementioned diagnostic techniques (both conventional PLC and molecular analysis) were included in the review.

\section{Results}

As of 2017, we found 51 studies dealing with PLC and molecular biological detection of free malignant cells in the peritoneum of GC patients (2, 4-54). Table I summarizes the principle features of the studies included in the review.

PLC. PL was collected by introducing, stirring and aspirating from the abdominal cavity an aliquot of saline solution ranging from $50 \mathrm{ml}(9,27)$ to $200 \mathrm{ml}(24,41)$. All the patients did not receive any neoadjuvant treatment and PLs were performed at the beginning of laparotomic gastrectomy (most works), laparoscopy surgery (conducted with curative or staging purpose) $(35-40,43)$, or paracentesis (only one case, 48). Conventional Papanicolaou staining was adopted in the vast majority of cases, followed by ordinary hematoxylin and eosin $(\mathrm{H} \& \mathrm{E})$ coloration $(9,11)$ and Giemsa stain $(17,28)$. Immunocytochemistry (ICC) was described only occasionally $(10,31,40,53)$. Sensitivity $(12.3 \%$ to $67 \%$ ) and specificity (94\% to $100 \%$ ) of PLC were clearly expressed as a percentage ratio only in 12 and 8 studies respectively (Table I).

Molecular detection of intraperitoneal free cancer cells. As for the molecular method, the mature messenger ribonucleic acid (messenger RNA, mRNA) of carcinoembryonic antigen (CEA) has been the target most commonly studied (41 articles) (2, 4-43) followed by 11 studies dealing with cytokeratin 20 (CK-20) mRNA $(18,25,29-32,36-39,42$, 49), 10 of which examined CEA mRNA concomitantly (18, 25, 29-32, 36-39, 49). Besides these, 23 additional mRNAs of other molecules have been occasionally investigated (11, $20-22,28,31,36,39,44-54)$. Concerning the type of molecular biological technique, qualitative RT-PCR has been the one most frequently adopted (27 studies), followed by quantitative RT-PCR (Q-RT-PCR) (24 studies). Recently, other ultra-rapid non-PCR tests, such as transcription-reverse transcription concerted reaction (TCR), and PCR-tests, such as reverse transcription loop-mediated isothermal amplification (RT-LAMP), have been successfully employed for genetic analysis $(33,34,43,54)$. Sensitivity and specificity of molecular examination were clearly expressed as a percentage ratio in 23 and 16 studies respectively (Table I). Sensitivity and specificity of CEA mRNA were respectively $38-100 \%$ and $7.3-100 \%$, whereas for CK-20 mRNA they were $25-64 \%$ and 80.3 to $94 \%$ (Table I).

\section{Discussion}

Major routes of metastatic spread in GC are direct infiltration of contiguous structures, hematogenous metastasis to the liver, regional lymph node metastasis, intraperitoneal dissemination, mesogastric pathway and intragastric exfoliation $(29,55)$. Of these, peritoneal dissemination is reported to be the most frequent pattern of metastasis and recurrence (32-54\%) in AGC (42). PM from GC results from a 2 step-process: the former is the exfoliation of free cancer cells from the serosal surface of the primary tumor into the peritoneal cavity, the latter is the attachment of cancer cells to a preferable intraperitoneal site (such as omentum, mesenterium and Douglas pouch) with subsequent growth and invasion of the abdominal cavity $(2,29,42)$. Furthermore, PM is also recognized as the most important independent prognostic factor for GC PR (29, 30). In fact, due to the development of PM even after R0 tumor resections, prognosis of AGC patients remain poor: to date, except for some individual experiences, no systemic or intraperitoneal treatment proved to effect a complete cure of AGC related-PM (56, 57). For this motive, the cytological examination of peritoneal lavage fluid has been adopted in clinical practice since 1999 by JGCA to detect free tumor cells floating in the abdominal cavity and predict PR $(3,24,41)$. However, despite an excellent specificity approximately of $100 \%$, conventional PLC through Papanicolaou or other classical stainings lack sensitivity (11.1 to $80 \%$ ) since PR is often observed in PLC-negative patients as well as in non-AGC cancers (that is not invading the serosal layer) (30, Table I). Such a disappointing constraint has been in part referred to the technical personal skills of the cytologist but, mostly, it has been reported that the manipulation of the tumor as well as surgical maneuvers (especially when the surgeons open the gastric wall or the lymphovascular vessels) can cause tumor spillage from gastric lumen to peritoneal cavity ensuing PM-PR (2, 58-64); in this sense, of interest, gastric lavage as well as PL might be helpful preventive methods to minimize the risk of spillage of GC cells and PR (2). To increase the sensitivity and reliability of cancer cell detection in PL, in the last two decades researchers employed ICC and molecular biological methods to detect epithelial cell-related targets (40). Compared to standard cytology, ICC with antibodies has been described improving the detection rate by $5 \%$ to $15 \%$; nevertheless, 
Table I. Main features of the 51 studies dealing with cytological and molecular detection of free intraperitoneal tumor cells in gastric cancer patients.

\begin{tabular}{|c|c|c|c|c|c|c|c|}
\hline Year & Reference & $\begin{array}{l}\text { Molecular } \\
\text { marker }\end{array}$ & $\begin{array}{l}\text { Molecular } \\
\text { technique }\end{array}$ & $\begin{array}{l}\text { Number of } \\
\text { Gc patients }\end{array}$ & $\begin{array}{l}\text { Main clinical } \\
\text { observation }\end{array}$ & Sensitivity & Specificity \\
\hline 1997 & 4 & CEA mRNA & RT-PCR & 48 & $\begin{array}{l}\text { First study on RT-PCR for } \\
\text { predicting GC PR }\end{array}$ & RT-PCR $>$ PLC & $\begin{array}{l}\text { PLC: } 100 \% \\
\text { RT-PCR: } 100 \%\end{array}$ \\
\hline 1998 & 5 & CEA mRNA & RT-PCR & 148 & $\begin{array}{l}\text { Correlation of PLC }+ \text { and } \\
\text { RT-PCR+ with OS }(p<0.002)\end{array}$ & n.a. & n.a. \\
\hline 1999 & 6 & CEA mRNA & $\begin{array}{l}\text { RT-PCR, } \\
\text { Q-RT-PCR }\end{array}$ & 199 & $\begin{array}{l}\text { Correlation of RT-PCR+ } \\
\text { with poor OS }(p<0.001)\end{array}$ & n.a. & n.a. \\
\hline 2000 & 7 & CEA mRNA & $\begin{array}{l}\text { RT-PCR, } \\
\text { Q-RT-PCR }\end{array}$ & 109 & $\begin{array}{c}\text { Correlation between Q-RT-PCR+ } \\
\text { and } \mathrm{pT}(p<0.01)\end{array}$ & n.a. & n.a. \\
\hline 2000 & 8 & CEA mRNA & RT-PCR & 43 & Correlation of RT-PCR+ with pT & $\begin{array}{c}\text { PLC }=33 \% \\
\text { RT-PCR }>38 \%\end{array}$ & n.a. \\
\hline 2001 & 9 & CEA mRNA & RT-PCR & 30 & $\begin{array}{l}\text { First study on omental } \\
\text { milky spots as PR }\end{array}$ & n.a. & $\begin{array}{l}\text { RT-PCR: } \\
\text { open issue }\end{array}$ \\
\hline 2001 & 10 & CEA mRNA & RT-PCR & 17 & Utility of both PLC and RT-PCR & $\begin{array}{l}\text { PLC: } 23 \% \\
\text { RT-PCR: } 63 \%\end{array}$ & n.a. \\
\hline 2001 & 11 & $\begin{array}{l}\text { CEA mRNA, } \\
\text { telomerase }\end{array}$ & $\begin{array}{l}\text { RT-PCR, } \\
\text { telomerase assay }\end{array}$ & n.f. & $\begin{array}{l}\text { Occurrence of PR } \\
\text { in PLC- patients }\end{array}$ & n.f. & n.f. \\
\hline 2001 & 12 & $\begin{array}{l}\text { CEA mRNA, } \\
\text { CK-19 mRNA, }\end{array}$ & RT-PCR & 7 & $\mathrm{CEA}>\mathrm{CK}-19$ as useful marker & n.a. & n.a. \\
\hline 2001 & 13 & CEA mRNA & RT-PCR & 230 & $\begin{array}{c}\text { PLC detects intraperitoneal cells } \\
\text { and predicts PR more sensitively } \\
\text { than RT-PCR }\end{array}$ & $\begin{array}{c}\text { PLC: } 46 \% \\
\text { RT-PCR: } 31 \% \\
\text { Combined: } 57 \%\end{array}$ & $\begin{array}{c}\text { PLC: } 94 \% \\
\text { RT-PCR: } 95 \%\end{array}$ \\
\hline 2002 & 14 & CEA mRNA & Q-RT-PCR & 90 & $\begin{array}{c}\text { RT-PCR predicts PR } \\
\text { more sensitively than PLC }\end{array}$ & $\begin{array}{l}\text { PLC: } 31 \% \\
\text { RT-PCR: } 77 \%\end{array}$ & $\begin{array}{l}\text { PLC: } 100 \% \\
\text { RT-PCR: } 94 \%\end{array}$ \\
\hline 2002 & 15 & CEA mRNA & RT-PCR & 86 & $\begin{array}{l}\text { RT-PCR as preferable method } \\
\text { to detect cells/PR }\end{array}$ & PLC $<$ RT-PCR & PLC $<$ RT-PCR \\
\hline 2003 & 16 & CEA mRNA & $\begin{array}{l}\text { RT-PCR } \\
\text { Q-RT-PCR }\end{array}$ & 65 & $\begin{array}{l}\text { PLC combined with Q-RT-PLC } \\
\text { as the most sensitive diagnostic } \\
\text { test }(p<0.05)\end{array}$ & $\begin{array}{c}\text { PLC: } 51 \% \\
\text { RT-PCR: } 49 \% \\
\text { QRT-PCR: } 42 \% \\
\text { Combined: } 70 \%\end{array}$ & n.a. \\
\hline 2003 & 17 & CEA mRNA & RT-PCR & 136 & $\begin{array}{l}\text { Advisable adjuvant therapy } \\
\text { for RT-PCR+ patients }\end{array}$ & n.a. & n.a. \\
\hline 2003 & 18 & $\begin{array}{l}\text { CEA mRNA, } \\
\text { CK-20 mRNA }\end{array}$ & Q-RT-PCR & 129 & $\begin{array}{l}\text { Multiplex Q-RT-PCR as } \\
\text { more reliable than PLC }\end{array}$ & PLC $<$ Q-RT-PCR & PLC $<$ Q-RT-PCR \\
\hline 2004 & 19 & CEA mRNA & Q-RT-PCR & 195 & $\begin{array}{l}\text { CEA mRNA as independent risk } \\
\text { factor for PR }(p=0.027)\end{array}$ & n.a. & n.a. \\
\hline 2004 & 20 & $\begin{array}{l}\text { CEA mRNA } \\
\text { L3-PP mRNA }\end{array}$ & Q-RT-PCR & 93 & Clinical utility of L3-PP mRNA & $\begin{array}{c}\text { PLC: } n . f . \\
\text { L3-PP: } 61 \% \\
\text { L3PP+CEA >L3PP }\end{array}$ & n.f. \\
\hline 2004 & 21 & $\begin{array}{l}\text { CEA mRNA, } \\
\text { L3-PP mRNA }\end{array}$ & Q-RT-PCR & 88 & $\begin{array}{l}\text { QRT-PCR combining L3-PP with } \\
\text { CEA as the most sensitive test for } \\
\text { detecting free intraperitoneal cells }\end{array}$ & $\begin{array}{c}\text { PLC: } 57 \% \\
\text { L3-PP: } 57 \% \\
\text { CEA: } 71 \% \\
\text { CEA+L3P: } 85.7 \%\end{array}$ & $\begin{array}{l}\text { PLC: } \text { n.a. } \\
\text { L3-PP: } 93 \% \\
\text { CEA: } 96 \% \\
\text { combined: } \text { n.a. }\end{array}$ \\
\hline 2004 & 22 & $\begin{array}{l}\text { CEA mRNA, } \\
\text { DDC mRNA }\end{array}$ & Q-RT-PCR & 114 & $\begin{array}{l}\text { Combination of CEA with DDC } \\
\text { improves the accuracy of } \\
\text { detecting free cancer cells }\end{array}$ & $\begin{array}{c}\text { CEA: } 73 \% \\
\text { DDC: } 87 \% \\
\text { CEA+DDC: } 93 \%\end{array}$ & n.f. \\
\hline 2005 & 23 & CEA mRNA & RT-PCR & 40 & $\begin{array}{l}\text { RT-PCR correlates with pT-TNM-PR } \\
\quad(p<0.001) \text { and } \mathrm{pN}(p=0.004)\end{array}$ & $\begin{array}{l}\text { PLC<RT-PCR } \\
\text { RT-PCR: } 82 \%\end{array}$ & n.a. \\
\hline 2005 & 24 & CEA mRNA & Q-RT-PCR & 80 & $\begin{array}{l}\text { CEA mRNA as independent prognostic } \\
\text { factor }(p=0.0130) \text {. No PLC analysis. }\end{array}$ & RT-PCR: $84.6 \%$ & RT-PCR: $87.7 \%$ \\
\hline 2005 & 25 & $\begin{array}{l}\text { CEA mRNA, } \\
\text { CK-20 mRNA }\end{array}$ & Q-RT-PCR & 230 & $\begin{array}{l}\text { High rates of false-positives with } \\
\text { CK-20; high specificity but limited } \\
\text { sensitivity of RT-PCR CK-20 }\end{array}$ & $\begin{array}{c}\text { PLC: n.a. } \\
\text { CEA: n.a. } \\
\text { CK-20: } 64 \% \\
\text { CK20+CEA:73\% }\end{array}$ & $\begin{array}{c}\text { PLC: } 95 \% \\
\text { CEA: } 90 \% \\
\text { CK-20: } 91 \% \\
\text { CK20+CEA: } 86 \%\end{array}$ \\
\hline 2006 & 26 & CEA mRNA & RT-PCR & 284 & $\begin{array}{l}\text { RT-PCR }+ \text { as independent risk } \\
\text { factor for cancer death }\end{array}$ & RT-PCR: $88.5 \%$ & RT-PCR: $81.6 \%$ \\
\hline
\end{tabular}


Table I. Continued

\begin{tabular}{|c|c|c|c|c|c|c|c|}
\hline Year & Referenc & $\begin{array}{l}\text { Molecular } \\
\text { marker }\end{array}$ & $\begin{array}{l}\text { Molecular } \\
\text { technique }\end{array}$ & $\begin{array}{l}\text { Number of } \\
\text { Gc patients }\end{array}$ & $\begin{array}{c}\text { Main clinical } \\
\text { observation }\end{array}$ & Sensitivity & Specificity \\
\hline 2006 & 27 & CEA mRNA & Q-RT-PCR & 65 & $\begin{array}{l}\text { Correlation of QRT-PCR+ with } \\
\text { pT and stage }\end{array}$ & $\begin{array}{c}\text { PLC: } 12.3 \% \\
\text { QRT-PCR: } 47.7 \%\end{array}$ & n.a. \\
\hline 2006 & 28 & $\begin{array}{l}\text { CEA mRNA, } \\
\text { IL-2 mRNA, } \\
\text { IL-10 mRNA }\end{array}$ & Q-RT-PCR & 110 & $\begin{array}{c}\text { CEA+ predicts poorer OS } \\
\text { than CEA- }(p=0.044)\end{array}$ & n.a. & n.a. \\
\hline 2007 & 29 & $\begin{array}{l}\text { CEA mRNA, } \\
\text { CK-20 mRNA }\end{array}$ & Q-RT-PCR & 131 & $\begin{array}{l}\text { PLC+ and QRT-PCR+ have lower } \\
\text { clinical significance in CRC than GC }\end{array}$ & n.a. & n.a. \\
\hline 2007 & 30 & $\begin{array}{l}\text { CEA mRNA, } \\
\text { CK-20 mRNA }\end{array}$ & Q-RT-PCR & 164 & $\begin{array}{l}\text { Combined QRT-PCR+ as } \\
\text { independent risk factor for } \\
\text { poor OS and DFS }(p<0.001)\end{array}$ & QRT-PCR $>$ PLC & n.a. \\
\hline 2007 & 31 & $\begin{array}{c}\text { CEA, CK-20, } \\
\text { FABP1, MUC2, } \\
\text { TFF1, TFF2, } \\
\text { MASPIN, GW112, } \\
\text { PRSS4, TACSTD1 }\end{array}$ & $\begin{array}{l}\text { Multiplex } \\
\text { RT-PCR }\end{array}$ & 179 & $\begin{array}{l}\text { RT-PCR+ and ICC+ as } \\
\text { portended poorer DFS than } \\
\text { RT-PCR- and ICC- }\end{array}$ & RT-PCR $>$ PLC & n.a. \\
\hline 2007 & 32 & $\begin{array}{l}\text { CEA mRNA, } \\
\text { CK-20 mRNA }\end{array}$ & Q-RT-PCR & 124 & $\begin{array}{l}\text { Worse OS for QRT-PCR+ than } \\
\text { QRT-PCR- patients }(p<0.001)\end{array}$ & $\begin{array}{c}\text { CEA: } 72.7 \% \\
\text { CK-20: } 54.6 \% \\
\text { combined: } 81.1 \%\end{array}$ & $\begin{array}{c}\text { CEA: } 82.7 \% \\
\text { CK-20: } 80.3 \% \\
\text { combined: } 79.7 \%\end{array}$ \\
\hline 2007 & 33 & CEA mRNA & TCR & n.f. & n.f. & n.f. & n.f. \\
\hline 2007 & 34 & CEA mRNA & $\begin{array}{l}\text { Q-RT-PCR, } \\
\text { TCR }\end{array}$ & 112 & $\begin{array}{l}\text { Correlation between TCR } \\
\text { and QRT-PCR }(p<0.0001)\end{array}$ & $\begin{array}{c}\text { PLC: } 61.5 \% \\
\text { TCR: } 84.6 \% \\
\text { QRT-PCR: } 92.3 \%\end{array}$ & $\begin{array}{c}\text { PLC: } 100 \% \\
\text { TCR: } 100 \% \\
\text { QRT-PCR: } 100 \%\end{array}$ \\
\hline 2007 & 35 & CEA mRNA & Q-RT-PCR & 156 & $\begin{array}{l}\text { QRT-PCR+ patients had } \\
\text { poorer survival }(p=0.0003)\end{array}$ & $\begin{array}{c}\text { PLC: } 61 \% \\
\text { QRT-PCR: } 79 \%\end{array}$ & n.a. \\
\hline 2008 & 36 & $\begin{array}{l}\text { CEA, CK-20, } \\
\text { Survivin, MUC2 }\end{array}$ & RT-PCR & 34 & $\begin{array}{l}\text { CEA mRNA resulted to be the } \\
\text { marker with best sensitivity } \\
\text { and specificity. Limit: } \\
\text { no marker mRNA } \\
\text { quantificationby RT-PCR. }\end{array}$ & $\begin{array}{l}\text { PLC: } 67 \% \\
\text { CEA: } 100 \% \\
\text { CK-20: } 60 \% \\
\text { Survivin: } 100 \% \\
\text { MUC2: } 40 \%\end{array}$ & $\begin{array}{c}\text { PLC: } 95 \% \\
\text { CEA: } 91 \% \\
\text { CK-20: } 94 \% \\
\text { Survivin: } 74 \% \\
\text { MUC2: } 100 \%\end{array}$ \\
\hline 2011 & 2 & CEA mRNA & Q-RT-PCR & 38 & $\begin{array}{l}\text { Gastric and peritoneal irrigation } \\
\text { prevents intraoperative cancer spillage }\end{array}$ & PLC $<$ Q-RT-PCR & n.a. \\
\hline 2014 & 37 & $\begin{array}{l}\text { CEA mRNA, } \\
\text { CK-20 mRNA }\end{array}$ & Q-RT-PCR & 104 & $\begin{array}{l}\text { Worse DFS for PCR+ } \\
\text { patients }(p=0.007)\end{array}$ & n.a. & n.a. \\
\hline 2014 & 38 & $\begin{array}{l}\text { CEA mRNA, } \\
\text { CK-20 mRNA }\end{array}$ & RT-PCR & 102 & $\begin{array}{l}\text { Ki-67 staining should be used to } \\
\text { distinguish viable } v s \text {. inactive } \\
\text { cancer cells }\end{array}$ & n.a. & n.a. \\
\hline 2014 & 39 & $\begin{array}{l}\text { CEA, CK-20, } \\
\text { MMP-7, TGF- } \beta 1, \\
\text { CA125 }\end{array}$ & Q-RT-PCR & 116 & $\begin{array}{c}\text { PLC had the lowest sensitivity but } \\
\text { the highest specificity }(p<0.001) \text {. } \\
\text { At multivariate analysis, } \\
\text { CEA and MMP-7 mRNAs } \\
\text { were found to be independent } \\
\text { prognostic factors }(p=0.028)\end{array}$ & $\begin{array}{c}\text { PLC: } 42.2 \% \\
\text { CEA: } 60 \% \\
\text { CK: } 20: 46.8 \% \\
\text { MMP-7: } 53.3 \% \\
\text { CA125: } 48.9 \% \\
\text { TGF- } \beta 1: 57.8 \% \\
\text { CEA+MMP7:71\% }\end{array}$ & $\begin{array}{c}\text { PLC: } 95.8 \% \\
\text { CEA: } 81.7 \% \\
\text { CK: } 20: 80.3 \% \\
\text { MMP-7: } 90.1 \% \\
\text { CA125: } 83.1 \% \\
\text { TGF- } \beta 1: 84.5 \% \\
\text { CEA+MMP7:74\% }\end{array}$ \\
\hline 2015 & 40 & CEA mRNA & 2-RT-PCR, OSNA & 75 & $\begin{array}{l}\text { Concordance of } 93.8 \% \\
\text { between PLC and OSNA }\end{array}$ & $\begin{array}{l}\text { PLC: } \text { n.a. } \\
\text { QRT-PCR: n.a. } \\
\text { OSNA: } 85 \%\end{array}$ & $\begin{array}{l}\text { PLC: } \text { n.a. } \\
\text { QRT-PCR: n.a. } \\
\text { OSNA: } 97.7 \%\end{array}$ \\
\hline 2016 & 41 & CEA mRNA & RT-PCR & 117 & $\begin{array}{l}\text { RT-PCR+ had shorter } \\
\text { DFS }(p=0.001)\end{array}$ & $\begin{array}{l}\text { PLC: } \text { n.a. } \\
\text { CEA: } 65 \%\end{array}$ & $\begin{array}{l}\text { PLC: } \text { n.a. } \\
\text { CEA: } 74.2 \%\end{array}$ \\
\hline 2017 & 42 & $\begin{array}{c}\text { CEA mRNA } \\
\text { CK-20 mRNA }\end{array}$ & RT-PCR & 132 & $\begin{array}{l}\text { RT-PCR+ did not correlate } \\
\text { with worse DFS }(p=0.39)\end{array}$ & $\begin{array}{l}\text { PLC: } n . a . \\
\text { CEA: n.a. } \\
\text { CK-20: } 25 \%\end{array}$ & $\begin{array}{l}\text { PLC: } \text { n.a. } \\
\text { CEA: } 7.3 \% \\
\text { CK-20: n.a. }\end{array}$ \\
\hline 2017 & 43 & CEA mRNA & TCR & 97 & $\begin{array}{l}\text { Association between reduced CEA } \\
\text { mRNA after induction chemotherapy } \\
\text { and longer survival }(p<0.001)\end{array}$ & n.a. & n.a. \\
\hline 1997 & 44 & E-cadherin mRNA & RT-PCR & 10 & Possible clinical utility & RT-PCR $>$ PLC & n.f. \\
\hline 1998 & $45 \mathrm{~T}$ & Trypsinogen-1 mRNA & RT-PCR & 42 & Possible clinical adoption & n.a. & n.a. \\
\hline 1999 & 46 & E-cadherin mRNA & RT-PCR & 52 & Potentially valuable for diffuse GC & n.a. & low \\
\hline
\end{tabular}


Table I. Continued

\begin{tabular}{|c|c|c|c|c|c|c|c|}
\hline Year & Reference & $\begin{array}{l}\text { Molecular } \\
\text { marker }\end{array}$ & $\begin{array}{l}\text { Molecular } \\
\text { technique }\end{array}$ & $\begin{array}{l}\text { Number of } \\
\text { Gc patients }\end{array}$ & $\begin{array}{c}\text { Main clinical } \\
\text { observation }\end{array}$ & Sensitivity & Specificity \\
\hline 2000 & 47 & telomerase & TRAP assay & 12 & possible clinical use & n.a. & n.a. \\
\hline 2001 & 48 & MMP-7 mCRNA & RT-PCR & 152 & $\begin{array}{c}\text { Both PLC and MMP-7 mRNA } \\
\text { are independent predictors } \\
\text { of PR }(p<0.001)\end{array}$ & $\begin{array}{c}\text { PLC: } 46 \% \\
\text { RT-PCR: } 33 \% \\
\text { combined: } 62 \%\end{array}$ & $\begin{array}{c}\text { PLC: } 95 \% \\
\text { RT-PCR: } 88 \% \\
\text { combined: } \text { n.a. }\end{array}$ \\
\hline 2004 & 49 & $\begin{array}{l}\text { CK-20, FABP1, } \\
\text { MUC2, TFF1, } \\
\text { TFF2 }\end{array}$ & RT-PCR & 99 & Advocating RT-PCR $v s$. Q-RT-PCR & n.a. & $\begin{array}{c}\text { multi-RT-PCR: } \\
\text { 91-100\% }\end{array}$ \\
\hline 2004 & 50 & Survivin mRNA & RT-PCR & 48 & $\begin{array}{l}\text { Promising help to future } \\
\text { therapeutic strategy }\end{array}$ & $\begin{array}{l}\text { RT-PCR+PLC: } \\
66.7 \% \text { (>PLC) }\end{array}$ & n.a. \\
\hline 2004 & 51 & $\begin{array}{l}\text { TGF } \beta, \text { IL-2, } \\
\text { IL-6 }\end{array}$ & Q-RT-PCR & 78 & $\begin{array}{l}\text { No significant difference in } \\
\text { expression between GC } \\
\text { and control patients }\end{array}$ & n.a. & n.a. \\
\hline 2005 & 52 & Heparanase mRNA & RT-PCR & 48 & Possible marker for detecting PM & RT-PCR $>$ PLC & n.a. \\
\hline 2014 & 53 & IL-17 mRNA & Q-RT-PCR & 114 & $\begin{array}{l}\text { Low expression as independent } \\
\text { predictive factor for } \\
\text { prognosis }(p=0.0098)\end{array}$ & n.a. & n.a. \\
\hline 2014 & 54 & CK-19 MRNA & RT-LAMP & 52 & $\begin{array}{l}\text { RT-LAMP+ significant } \\
\text { for PR }(p<0.005)\end{array}$ & RT-LAMP $>$ PLC & n.a. \\
\hline
\end{tabular}

Q-RT-PCR: Real-time quantitative PCR; CEA: carcinoembryonic antigen; PR: peritoneal recurrence; PLC: peritoneal lavage cytology; CK: cytokeratin; L3-PP: L-3 phosphoserine phosphatase; DDC: dopa decarboxylate; IL: interleukin; OS: overall survival; DFS: disease free survival; TCR: transcription-reverse transcription concerted reaction; MMP: matrix metalloproteinase; OSNA: one-step nucleic acid amplification; TRAP: telomeric repeat amplification protocol; RT-LAMP: reverse transcription-loop mediated isothermal amplification; CRC: colorectal cancer; ICC: immunocytochemical cytology; n.a.: not assessed; n.f.: not findable.

PM can still occur in PLC-ICC negative cases $(36,40)$. Molecular detection with qualitative or quantitative RTPCR have identified potent molecular markers of various target genes such as transcripts of CEA, CK-20, MMP-7, heparanase and many other molecules (Table I). In most cases, if not all, molecular results (especially those on CEA mRNA) have proved to be superior to cytological ones in sensitivity and prognostic prediction for survival $(10,14$, 27, 34-36, 39); in particular, GC patients expressing molecular positive tests, have been identified to benefit from more aggressive adjuvant treatment including intraperitoneal chemotherapy with paclitaxel (43). Concerning specificity, on the other hand, both methods have often achieved $100 \%(4,34)$ and PLC not infrequently was superior $(14,25,39,48)$. Currently however, yet promising, the detection molecular methods are not applied for routine use everywhere: sometimes, in fact, they provided controversial results (such as false-positive cases with mRNAs released by lymphocytes and sane mesothelial cells and other false-positives results due to isolated tumor cells -that is clinically insignificant cells without metastatic potential- and not to micrometastases -the utter active metastatic cells-) and, most of all, they are time-consuming, labor-intensive and expensive $(1,32,24,40)$. With this respect, testing novel ultra-rapid molecular methods (such as OSNA, TCR and LAMP) $(33,34,40,43,54)$ with innovative targets isolated not only from PLs but also from blood or tissue specimens of AGC patients $(65,66)$ could surmount some temporal and costly limitations of the current genetic techniques. Furthermore, they could provide an opportunity to perform reliable tailor-made surgery for GC as a common procedure in general hospitals (54).

\section{Conclusion}

Detecting free tumor cells in peritoneal lavage fluid of AGC patients is of paramount importance in order to predict PR, assess a more accurate prognosis and select cases for more intensive treatment. So far, given the imperfection of each method, employment of both cytology and molecular method seem to be mandatory for achieving this aim.

\section{Conflicts of Interest}

The Authors declare no conflicts of interest.

\section{References}

1 Fujiwara Y, Doki Y, Taniguchi H, Sohma I, Takiguchi S, Miyata $\mathrm{H}$, Yamasaki M and Monden M: Genetic detection of free cancer cells in the peritoneal cavity of the patient with gastric cancer: present status and future perspectives. Gastric Cancer 10: 197204, 2007. 
2 Han TS, Kong SH, Lee HJ, Ahn HS, Hur K, Yu J, Kim WH and Yang HK: Dissemination of free cancer cells from the gastric lumen and from perigastric lymphovascular pedicles during radical gastric cancer surgery. Ann Surg Oncol 18: 2818-2825, 2011.

3 La Torre M, Ferri M, Giovagnoli MR, Sforza N, Cosenza G, Giarnieri E and Ziparo V: Peritoneal wash cytology in gastric carcinoma. Prognostic significance and therapeutic consequences. Eur J Surg Oncol 36: 982-986, 2010.

4 Nakanishi H, Kodera Y, Torii A, Hirai T, YamamuraY, Kato T, Kito $\mathrm{T}$ and Tatematsu $\mathrm{M}$ : Detection of carcinoembryonic antigen-expressing free tumor cells in peritoneal washes from patients with gastric carcinoma by polymerase chain reaction. Jpn J Cancer Res 88: 687-692, 1997.

5 Kodera Y, Nakanishi H, Yamamura Y, Shimizu Y, Torii A, Hirai T, Yasui K, Morimoto T, Kato T, Kito T and Tatematsu M: Prognostic value and clinical implications of disseminated cancer cells in the peritoneal cavity detected by reverse transcriptase-polymerase chain reaction and cytology. Int $\mathrm{J}$ Cancer 79: 429-433, 1998.

6 Nakanishi H, Kodera Y, Yamamura Y, Kuzuya K, Nakanishi T, Ezaki T and Tatematsu M: Molecular diagnostic detection of free cancer cells in the peritoneal cavity of patients with gastrointestinal and gynecologic malignancies. Cancer Chemother Pharmacol 43: 32-36, 1999.

7 Nakanishi H, Kodera Y, Yamamura Y, Ito S, Ezaki $\mathrm{T}$ and Tatematsu M: Rapid quantitative detection of carcinoembryonic antigen-expressing free tumor cells in the peritoneal cavity of gastric-cancer patients with real-time RT-PCR on the lyghtcycler. Int J Cancer 89: 411-417, 2000.

$8 \mathrm{Xu} \mathrm{H}$, Wang $\mathrm{J}$ and Wang $\mathrm{Z}$ : The value of CEA mRNA, CEA protein and cytology of peritoneal washings in the prediction of metastasis of gastric cancer. Zhonghua Zhong Liu Za Zhi 22: 4547, 2000.

9 Sakakura C, Hagiwara A, Shirasu M, Yasouka R, Fujita Y, Nakanishi M, Aragane H, Masuda K, Shimomura K, Abe T and Yamagishi H: Polymerase chain reaction for detection of carcinoembryonic antigen-expressing tumor cells on milky spots oft he greater omentum in gastric cancer patients: a pilot study. Int J Cancer (Pred Oncol) 95: 286-289, 2001.

10 Broll R, Weschta M, Windhoevel U, Berndt S, Schwandner O, Roblick U, Schiedeck TH, Schimmelpenning H, Bruch HP and Duchrow M: Prognostic significance of free gastrointestinal tumor cells in peritoneal lavage detected by immunocytochemistry and polymerase chain reaction. Langenbecks Arch Surg 386: 285-292, 2001.

11 Tsujjtani S and Kaibara N: Clinical significance of molecular biological detection of micrometastases in gastric carcinoma. Nihon Geka Gakkai Zasshi 102: 741-744, 2001.

12 Schmidt P, Thiele M, Rudroff C, Vaz A, Schilli M, Friedrich K and Scheele J: Detection of tumor cells in peritoneal lavages from patients with gastrointestinal cancer by multiplex reverse transcriptase PCR. Hepatogastroenterology 48: 1675-1679, 2001.

13 Yonemura Y, Endou Y, Fujimura T, Fushida S, Bandou E, Kinoshita E Sugiyama K, Sawa T, Kim BS and Sasaki T: Diagnostic value of preoperative RT-PCR-based screening method to detect carcinoembryonic antigen-expressing free cancer cells in the peritoneal cavity from patients with gastric cancer. ANZ J Surg 71: 521-528, 2001.

14 Kodera Y, Nakanishi H, Ito S, Yamamura Y, Kanemitsu Y, Shimizu $Y$, Hirai $T$, Yasui $K$, Kato $T$ and Tatematsu $M$ :
Quantitative detection of disseminated cancer cells in the greater omentum of gastric carcinoma patients with real-time RT-PCR: a comparison with peritoneal lavage cytology. Gastric Cancer 5: 69-76, 2002.

15 Fujii S, Kitayama J, Kaisaki S, Sasaki S, Seto Y, Tominaga O, Tsuno N, Umetani N, Yokota H, Kitamura K, Tsuruo $\mathrm{T}$ and Nagawa $\mathrm{H}$ : Carcinoembryonic antigen mRNA in abdominal cavity as a useful predictor or peritoneal recurrence of gastric cancer with serosal exposure. J Exp Clin Cancer Res 21: 547-553, 2002.

16 To EM, Chan WY, Chow C, Ng EK and Chung SC: Gastric cancer cell in peritoneal washing: cytology versus RT-PCR for CEA transcripts. Diagn Mol Pathol 12: 88-95, 2003.

17 Tokuda K, Natsugoe S, Nakajo A, Miyazono F, Ishigami S, Hokita S, Takao S, Eizuru Y and Aikou T: Clinical significance of CEA-mRNA expression in peritoneal lavage fluid from patients with gastric cancer. Int J Mol Med 11: 79-84, 2003.

18 Sugita Y, Fujiwara Y, Taniguchi H, Mori T, Ishii T, Niwa H, Okada Y, Takiguchi S, Yasuda T, Yano $\mathrm{M}$ and Monden $\mathrm{M}$ : Quantitative molecular diagnosis of peritoneal lavage fluid for prediction of peritoneal recurrence in gastric cancer. Int $\mathrm{J}$ Oncol 23: 1419-1423, 2003.

19 Oyama K, Terashima M, Takagane A and Maesawa C: Prognostic significance of peritoneal minimal residual disease in gastric cancer detected by reverse transcription-polymerase chain reaction. Br J Surg 91: 435-443, 2004.

20 Shimomura K, Ohgaki M, Kakihara N, Matsumura H, Miyata K, Iizuka W, Shimotsuma M, Takenaka A, Sakakura C, Hagiwara $\mathrm{A}$ and Yamagishi $\mathrm{H}$ : Nobel genes improve accuracy in detection of peritoneal micro-metastasis of gastric cancer to decide indication for chemotherapy. Gan To Kagaku Ryoho 31: 19121914, 2004

21 Shimomura K, Sakakura C, Takemura M, Takagi T, Fukuda K, Kin S, Nakase Y, Miyagawa K, Ohgaki M, Fujiyama J, Fujita Y, Nakanishi M, Hagiwara A, Shirane M, Okazaki Y, Hayashizaki $\mathrm{Y}$ and Yamagishi $\mathrm{H}$ : Combination of L-3-phosphoserine phosphatase and CEA using real-time RT-PCR improves accuracy in detection of peritoneal micrometastasis of gastric cancer. Anticancer Res 24: 1113-1120, 2004

22 Sakakura C, Takemura M, Miyagawa K, Shimomura K, Kin S, Nakase Y, Kuriu Y, Nakashima S, Yoshikawa T, Ueda Y, Fujiyama J, Sonoyama T, Okazaki Y, Hayashizaki Y, Hagiwara A and Yamagishi H: Utility of dopa decarboxylase as a novel marker fort he detection of peritoneal micro-metastases of gastric cancer with realtime RT-PCR. Gan To Kagaku Ryoho 31: 1906-1908, 2004.

23 Wang JY, Lin SR, Lu CY, Chen CC, Wu DC, Chai CY, Chen FM, Hsieh JS and Huang TJ: Gastric cancer cell detection in peritoneal lavage: RT-PCR for carcinoembryonic antigen transcripts versus the combined cytology with peritoneal carcinoembryonic antigen levels. Cancer Lett 223: 129-135, 2005.

24 Ito S, Kakanishi H, Kodera Y, Mochizuki Y, Tatematsu M and Yamamura Y: Prospective validation of quantitative CEA mRNA detection in peritoneal washes in gastric carcinoma patients. $\mathrm{Br}$ J Cancer 93: 986-992, 2005.

25 Kodera Y, Nakanishi H, Ito S, Yamamura Y, Fujiwara M, Koike M, Hibi K, Ito K, Tatematsu $\mathrm{M}$ and Nakao A: Prognostic significance of intraperitoneal cancer cells in gastric carcinoma: detection of cytokeratin 20mRNA in peritoneal washes, in addition to detection of carcinoembryonic antigen. Gastric Cancer 8: 142-148, 2005 
26 Kodera $\mathrm{Y}$, Nakanishi $\mathrm{H}$, Ito $\mathrm{S}$, Mochizuki $\mathrm{Y}$, Ohashi N, Yamamura Y, Fujiwara M, Koike M, Tatematsu M and Nakao A: Prognostic significance of intraperitoneal cancer cells in gastric carcinoma: analysis of real time reverse transcriptase-polymerase chain reaction after 5 years of followup. J Am Coll Surg 202: 231-236, 2006

27 Zhang YS, Xu J, Luo GH, Wang RC, Zhu J, Zhang XY, NilssonEhle $\mathrm{P}$ and $\mathrm{Xu} \mathrm{N}$ : Detection of carcinoembryonic antigen mRNA in peritoneal washes from gastric cancer patients and ist clinical significance. World J Gastroenterol 12: 1408-1411, 2006.

28 Fukumoto Y, Ikeguchi M, Matsumoto S, Inoue M, Osaki T, Fukuda K, Saito H, Tatebe S and Tsujitani S: Detection of cancer cells and gene expression of cytokines in the peritoneal cavity in patients with gastric cancer. Gastric Cancer 9: 271-276, 2006.

29 Hara M, Nakanishi H, Jun Q, Kanemitsu Y, Ito S, Mochizuki Y, Yamamura Y, Kodera Y, Tatematsu M, Hirai T and Kato T: Comparative analysis of intraperitoneal minimal free cancer cells between colorectal and gastric cancer patients using quantitative RT-PCR: possible reason for rare peritoneal recurrence in colorectal cancer. Clin Exp Metastasis 24: 179-189, 2007.

30 Tamura N, Inuma $\mathrm{H}$ and Takada T: Prospective study of the quantitative carcinoembryonic antigen and cytokeratin $20 \mathrm{mRNA}$ detection in peritoneal washes to predict peritoneal recurrence in gastric carcinoma patients. Oncol Rep 17: 667-672, 2007.

31 Mori K, Suzuki T, Uozaki H, Nakanishi H, Ueda T, Matsuno Y, Kodera Y, Sakamoto H, Yamamoto N, Sasako M, Kaminishi M and Sasaki H: Detection of minimal gastric cancer cells in peritoneal washings by focused microarray analysis with multiple markers: clinical implications. Ann Surg Oncol 14: 1694-1702, 2007.

32 Katsuragi K, Yashiro M, Sawada T, Osaka H, Ohira M and Hirakawa K: prognostic impact of PCR-based identification of isolated tumour cells in the peritoneal lavage fluid of gastric cancer patients who underwent a curative $\mathrm{R} 0$ resection. Br J Cancer 97: 550-556, 2007.

33 Hanada H, Fujiwara Y, Sohma I, Nakamura Y, Takeoka K, Iwatani $\mathrm{Y}$ and Hidaka Y: Peritoneal lavage diagnosis with TRC (transcription reverse transcription concerted reaction) system for prediction of peritoneal recurrence in gastric cancer. Rinsho Byori 55: 1002-1007, 2007.

34 Ohashi N, Nakanishi H, Kodera Y, Ito S, Mochizuki Y, Koike M, Fujiwara M, Yamamura Y, Tatematsu M, Nakao A and Kato $\mathrm{T}$ : Intraoperative quantitative detection of CEA mRNA in the peritoneal lavage of gastric cancer patients with transcription reverse-transcription concerted (TRC) method. A comparative study with real-time quantitative RT-PCR. Anticancer Res 27: 2769-2778, 2007.

35 Wong J, Kelly KJ, Mittra A, Gonen M, Allen P, Fong Y and Coit D: RT-PCR increases detection of submicroscopic peritoneal metastases in gastric cancer and has prognostic significance. $\mathrm{J}$ Gastrointest Surg 16: 889-896, 2012.

36 Dalal KM, Woo Y, Kelly K, Galanis C, Gonen M, Fong Y and Corr DG: Detection of micrometastases in peritoneal washings of gastric cancer patients by the reverse transcriptase polymerase chain reaction. Gastric Cancer 11: 206-213, 2008.

37 Takata A, Kurokawa Y, Fujiwara Y, Nakamura Y, Takahashi T, Yamasaki M, Miyata H, Nakajima K, Takiguchi S, Mori M and Doki Y: Prognostic value of CEA and CK20 mRNA in the peritoneal lavage fluid of patients undergoing curative surgery for gastric cancer. World J Surg 38: 1107-1111, 2014.
38 Takebayashi K, Muarat S, Yamamoto H, Ishida M, Yamaguchi T, Kojima M, Shimizu T, Shiomi H, Sonoda H, Naka S, Mekata E, Okabe $\mathrm{H}$ and Tani T: Surgery-induced peritoneal cancer cells in patients who have undergone curative gastrectomy for gastric cancer. Ann Surg Oncol 21: 1991-1997, 2014.

39 Li Z, Zhang D, Zhang H, Miao Z, Tang Y, Sun G and Dai D: Prediction of peritoneal recurrence by the mRNA level of CEA and MMP-7 in peritoneal lavage of gastric cancer patients. Tumor Biol 35: 3463-3470, 2014.

40 Nakabayashi K, Uraoka T, Shibuya M, Matsuura N and Tsujimoto M: Rapid detection of CEA mRNA in peritoneal washes using one-step nucleic acid amplification $\left(\mathrm{OSNA}^{\circledR}\right)$ for gastric cancer patients. Clin Chim Acta 439: 137-142, 2015.

41 Chae HD and Kim ICH: Prognostic significance of CEA expression by RT-PCR in peritoneal wash from patients with gastric cancer: result of a 5-year follow-up after curative resection. Scand J Gastroenterol 51: 956-960, 2016.

42 Kim YJ, Chung WC, Choi S, Jung YD, Lee J, Chae SY, Jun KH and Chin HM: The detection of messenger RNA for carcinoembryonic antigen and cytokeratin 20 in peritoneal washing fluid in patients with advanced gastric cancer. Korean J Gastroenterol 69: 220-225, 2017.

43 Yamaguchi H, Satoh Y, Ishigami H, Kurihara M, Yatomi Y and Kitayama J: Peritoneal lavage CEA mRNA levels predict conversion gastrectomy outcomes after induction chemotherapy with intraperitoneal paclitaxel in gastric cancer patients with peritoneal metastasis. Ann Surg Oncol 24: 3345-3352, 2017.

44 Fujimura T, Yonemura Y, Ninomiya I, Miwa K, Miyazaki I, Endo Y, Ishikawa N, Obata T, Tanaka M and Sasaki T: Oncol Rep 4: 1015-1019, 1997.

45 Fujimura T, Ohta T, Kitagawa H, Fushida S, Nishimura GI, Yonemura Y, Elnemr A, Miwa K and NakanumaY: Trypsinogen expression and early detection for peritoneal dissemination in gastric cancer. J Surg Oncol 69: 71-75, 1998.

46 Schuhmacher C, Becker KF, Reich U, Schenk U, Mueller J, Siewert JR and Höfler H: Rapid detection of mutated E-cadherin in peritoneal lavage specimens from patients with diffuse-type gastric carcinoma. Diagn Mol Pathol 8: 66-70, 1999.

47 Mori N, Oka M, Hazama S, Iizuka N, Yamamoto K, Yoshino S, Tangoku A, Noma T and Hirose K: Detection of telomerase activuty in peritoneal lavage fluid from patients with gastric cancer using immunomagnetic beads. Br J Cancer 83: 1026-1032, 2000.

48 Yonemura Y, Fujimura T, Ninomiya I, Kim BS, Bandou E, Sawa T, Kinoshita K, Endo Y, Sugiyama K and Sasaki T: Prediction of peritoneal micrometastasis by peritoneal lavaged cytology and reverse transcriptase-polymerase chain reaction for matrix metalloproteinase-7 mRNA. Clin Cancer Res 7: 1647-1653, 2001.

49 Mori K, Aoyagi K, Ueda T, Danjoh I, Tsubosa Y, Yanagihara K, Matsuno Y, Sasako M, Sakamoto H, Mafune Ki, Kaminishi M, Yoshida T, Terada M and Sasaki H: Highly specific marker genes for detecting minimal gastric cancer cells in cytology negative peritoneal washings. Biochem Biophys Res Commun 313: 931-937, 2004.

50 Wang ZN, Xu HM, Jiang L, Zhou X, Lu C and Zhang X: Expression of survivin mRNA in peritoneal lavage fluid from patients with gastric carcinoma. Chin Med J (Engl) 117: 1210$1217,2004$.

51 Ikeguchi M, Matsumoto S, Murakami D, Kanaji S, Ohro S, Maeta Y, Tatebe S, Kondo A, Tsujitani S and Kaibara N: Gene 
expression levels of cytokines in peritoneal washings from patients with gastric cancer. Tumour Biol 25: 117-121, 2004.

52 Wang Z, Zhang X, Xu H, Zhou X, Jiang L and Lu C: Detection of peritoneal micrometastasis by reverse transcriptasepolymerase chain reaction for heparanase mRNA and cytology in peritoneal wash samples. J Surg Oncol 90: 59-65, 2005.

53 IIda $T$, Iwahashi $M$, Katsuda $M$, Ishida $K$, Nakamori $M$, Nakamura M, Naka T, Ojima T, Ueda K, Hayata K, Yasuoka H and Yamaue H: Prognostic significance of IL-17 mRNA expression in peritoneal lavage in gastric cancer patients who underwent curative resection. Oncol Rep 31: 605-612, 2014.

54 Yoneda A, Taniguchi K, Torashima Y, Susumu S, Kanetaka K, Kuroki $\mathrm{T}$ and Eguchi S: The detection of gastric cancer cells in intraoperative peritoneal lavage using the reverse transcriptionloop mediated isothermal amplification method. J Surg Res 187: e1-6, 2014.

55 Virgilio E, D'Antonio C and Balducci G: Mesogastrium recurrence as expression ofthe fifth metastatic route of gastric cancer. Med Hypotheses 82: 403-404, 2014.

56 Masuishi T, Kadowaki S, Kondo M, Komori A, Sugiyama K, Mitani S, Honda K, Narita Y, Taniguchi H, Ura T, Ando M, Mishim H and Muro K: FOLFOX as first-line therapy for gastric cancer with severe peritoneal metastasis. Anticancer Res 37: 7037-7042, 2017.

57 Kanazawa Y, Fujita I, Kakinuma D, Arai H, Matsuno K, Shimoda T, Ko K, Kato S and Uchida E: Initial experience with Nab-Paclitaxel for patients with advanced gastric cancer: safety and efficacy. Anticancer Res 37: 2715-2720, 2017.

58 Virgilio E, Giarnieri E, Montagnini M, D’Urso R, Proietti A, Mesiti A, Giovagnoli MR, Mercantini P, Cavallini M and Balducci G: Analyzing gastric lavage of gastric cancer patients: a prospective observational study on cytopathology and determination of intragastric CEA, Ca 19.9, Ca 72.4 and $\mathrm{Ca} 50$. Acta Cytol 60: 161-166, 2016.

59 Virgilio E, Giarnieri E, Montagnini M, D’Urso R, Proietti A, Mesiti A, Giovagnoli MR, Mercantini P, Cavallini M and Balducci G: Detection of cancer cells and tumor markers in gastric lavage of patients with gastric cancer: do these findings have a clinicopathological significance and oncological implication? Med Hypotheses 94: 1-3, 2016.

60 Virgilio E, Giarnieri E, Giovagnoli MR, Montagnini M, Proietti A, D’Urso R, Nigri G, Mercantini P, Ramacciato G, Cavallini M and Balducci G: Presence of cancer cells in gastric lavage of gastric cancer patients as an indicator of advanced disease, predictor of tumour aggressive phenotype and independent prognostic factor for poor survival: The endoluminal metastatic pathway of gastric cancer and GL0/GL1 classification. Cytopathology 29: 41-49, 2018.
61 Virgilio E, Giarnieri E, Giovagnoli MR, Montagnini M, Proietti A, D’Urso R, Mercantini P, Balducci G and Cavallini M: Early gastric cancer exfoliating into gastric lavage (GL1 EGC) shows a more aggressive behavior and poorer survival compared to the non-exfoliative counterpart (GL0 EGC). Anticancer Res 37: 4199-4203, 2017.

62 Virgilio E, Proietti A, D’Urso R, Cardelli P, Giarnieri E, Montagnini M, Giovagnoli MR, Mercantini P, Balducci G and Cavallini M: Measuring intragastric tumor markers in gastric cancer patients: a systematic literature review on significance and reliability. Anticancer Res 37: 2817-2821, 2017.

63 Virgilio E, Balducci G, Mercantini P, Ferri M, Bocchetti T, Caterino S, Salvi PF, Ziparo V and Cavallini M: Reconstruction after distal gastrectomy for gastric cancer: Billroth 2 or RouxEn-Y procedure? Anticancer Res 37: 5595-5602, 2017.

64 Virgilio E, Balducci G, Mercanini P, Giarnieri E, Giovagnoli MR, Montagnini M, Proietti A, D'Urso R and Cavallini M: Utility of nasogastric tube for medical and surgical oncology of gastric cancer: a prospective institutional study on a ne wand precious application of an old and economic device. Anticancer Res 38: 433-439, 2018.

65 Katayama Y, Oshima T, Sakamaki K, Aoyama T, Sato T, Masudo K, Shiozawa M, Yoshikawa T, Rino Y, Imada T and Masuda M: Clinical significance of INHBA gene expression in patients with gastric cancer who receive curative resection followed by adjuvant S-1 chemotherapy. In Vivo 31: 565-571, 2017.

66 Tahara T, Tahara S, Horiguchi n, Kawamura T, Okubo M, Ishizuka T, Yamada H, Yoshida D, Ohmori T, Maeda K, Komura N, Ikuno H, Jodai Y, Kamano T, Nagasaka M, Nakagawa Y, Tuskamoto T, Urano M, Shibata T, Kuroda M and Ohmiya N: Telomere length in leukocyte DANN in gastric cancer patients and ist association with clinicopathological features and prognosis. Anticancer Res 37: 1997-2001, 2017.
Received December 15, 2017

Revised January 27, 2018

Accepted January 29, 2018 